\title{
Conclusions générales
}

Des progrès substantiels ont été réalisés au cours des dernières années dans le traitement de l'infection ostéo-articulaire:

Sur le plan des connaissances : la situation particulière des bactéries au contact d'un corps étranger a été bien mise en évidence par les travaux de Gristina. La production par les microorganismes de glycocalix ainsi que les biofilms (fibronectine, fibrine, laminine et autres) déposés sur les implants modifient l'influence des agents antimicrobiens.

Le traitement chirurgical reste la clef du succès. Il vise à éliminer les tissus dévitalisés et infectés, à réduire le nombre de bactéries et à améliorer la perfusion des zones infectées.

Sur ce plan, un apport récent majeur est l'utilisation de plus en plus fréquente et précoce de plasties musculaires, libres ou pédiculées, pour combler les pertes de substance osseuse et apporter un lit vasculaire aux zones infectées et cicatricielles.

La technique des transports osseux d'llisarov permet de résoudre des problèmes de perte de substance cutanée en même temps qu'osseuse.

L'utilisation de "spacers", billes ou blocs de ciment, généralement imprégnés d'antibiotiques, a permis de placer de nouvelles prothèses totales quelque temps après leur ablation.

L'apport local d'antibiotiques obéit à la logique de porter la substance là où elle est utile. L'administration par pompe interne (Perry 1989) ou externe (Meani 1993) ouvre des voies nouvelles pour les traitements de longue durée. De nouveaux supports sont disponibles : collagène (Braun 1985) avec ses formes nouvelles, fibrine, os spongieux, polyméthacrylate de méthyle traité pour changer la diffusion des antibiotiques (polymérisation sous vide, addition de dextran), ciment biodégradable (Gerhardt 1988).

Les antiseptiques ou substances voisines sont restés à la traîne. La taurolidine proposée par Pfirrmann en 1985 a fait ses preuves chez un groupe restreint d'utilisateurs mais n'est pas introduite dans de nombreux pays et subit, dès lors, les conséquences d'une diffusion trop limitée. Baltzer a publié en 1989 les résultats d'une biguanide "GX" étudiée entre autres par Willenegger ${ }^{1}$. Cette substance est apparentée à la chlorhexidine et me paraît avoir un certain avenir en raison de l'absence de toxicité et d'un spectre d'action très large. On ne connaît pas de résistance

\footnotetext{
${ }^{1}$ Le GX est commercialisé sous la dénomination "Lavasept"
}

bactérienne à cette substance.

Dans le contexte actuel du développement des résistances aux antibiotiques au fur et à mesure de leur mise en action, on ne peut qu'espérer l'apparition d'une chimiothérapie locale efficace.

Pour terminer, les problèmes majeurs qui nous restent à affronter me paraissent les suivants :

Les arthrites dépendent encore du traitement antibiotique par voie générale. Les antibiotiques locaux ne peuvent être prescrits à haute dose en raison de leur résorption, les antiseptiques sont toxiques pour le cartilage. Les cas vus tardivement finissent encore trop souvent avec d'importantes séquelles fonctionnelles.

Les souches de staphylocoques méthicillino-résistantes deviennent un inquiétant problème d'hygiène hospitalière dans les unités d'orthopédie septique.

Certaines mycobactéries responsables d'ostéites sont résistantes aux tuberculostatiques connus.

La durée du traitement antibiotique, par voie générale, des infections osseuses reste arbitraire. Les durées de prescriptions par voie veineuse, six semaines souvent, exigent des hospitalisations coûteuses. Le paramètre objectif qui permettrait de mettre fin au traitement nous manque. 